\title{
List of Figures in the Introduction
}

Frontispiece: Engraving of a female figure by Cornelis Bos [1539] (see Cat. 8) (MAK-Österreichisches Museum für angewandte Kunst, Vienna).

1. Johannes de Ketham, Fasciculo di medicina, Venice, J. \& G. de Gregoriis, 1493 [i.e. 1494]. Anatomy lesson (fol. f2v), woodcut (from Fasciculo di medicina, repr. with intro. by C. Singer, Florence, 1925; photo: Wellcome Library, London).

2. Andreas Vesalius, De humani corporis fabrica, Basel, J. Oporinus, 1543. Title-page, woodcut (Wellcome Library, London).

3. Andreas Vesalius, De humani corporis fabrica, Basel, J. Oporinus, 1543. Portrait of Vesalius (fol. *6v), woodcut (Wellcome Library, London).

4. Johannes de Ketham, Fasciculus medicinae, Venice, J. and G. de Gregoriis, 1495. Figura matricis (fol. b2v), woodcut (Wellcome Library, London).

5. Johannes de Ketham, Fasciculus medicinae, Venice, J. and G. de Gregoriis, 1495. Zodiac man (fol. b2r), woodcut (Wellcome Library, London).

6. Johannes de Ketham, Fasciculus medicinae, Venice, J. and G. de Gregoriis, 1495. Wound man (fol. c2r), woodcut (Wellcome Library, London).

7. Johannes de Ketham, Fasciculus medicinae, Venice, J. and G. de Gregoriis, 1495. Bloodletting man (fol. a4r), woodcut (Wellcome Library, London).

8. Jacopo Berengario da Carpi, Commentaria, cum amplissimis additionibus super anatomia Mundini . . . , Bologna, $\mathrm{H}$. de Benedictis, 1521. Figura matricis (fol. $\operatorname{ccxxv}^{v}$ ), woodcut (Wellcome Library, London).

9. Jacopo Berengario da Carpi, Commentaria, cum amplissimis additionibus super anatomia Mundini . . . , Bologna, H. de Benedictis, 1521. Figura matricis (fol. cccxxvi ${ }^{\mathrm{r}}$ ), woodcut (Wellcome Library, London).

10. Jacopo Berengario da Carpi, Commentaria, cum amplissimis additionibus super anatomia Mundini ... ., Bologna, H. de Benedictis, 1521. Muscle man (fol. lxxxiir), woodcut (Wellcome Library, London). 
11. Charles Estienne, De dissectione partium corporis humani libri tres, Paris, S. de Colines, 1545. Skeleton (p. 43), woodcut (Wellcome Library, London).

12. Charles Estienne, De dissectione partium corporis humani libri tres, Paris, S. de Colines, 1545. Figure with a section of the head (p. 242), woodcut (Wellcome Library, London).

13. Charles Estienne, De dissectione partium corporis humani libri tres, Paris, S. de Colines, 1545. Female anatomical figure (p. 260), woodcut (Wellcome Library, London).

14. Charles Estienne, De dissectione partium corporis humani libri tres, Paris, S. de Colines, 1545. Female anatomical figure (p. 279), woodcut (Wellcome Library, London).

15. Charles Estienne, De dissectione partium corporis humani libri tres, Paris, S. de Colines, 1545. Female anatomical figure (p. 281), woodcut (Wellcome Library, London).

16. Charles Estienne, De dissectione partium corporis humani libri tres, Paris, S. de Colines 1545. Female anatomical figure (p. 271), woodcut (Wellcome Library, London).

17. Giovanni Jacopo Caraglio after Perino del Vaga, Venere e Amore, part of the series, Gli amori degli dei, engraving (cliché Bibliothèque nationale de France, Paris).

18. Charles Estienne, De dissectione partium corporis humani libri tres, Paris, S. de Colines, 1545. Female anatomical figure (p. 267), woodcut (Wellcome Library, London).

19. Charles Estienne, De dissectione partium corporis humani libri tres, Paris, S. de Colines, 1545. Female figure (p. 287), woodcut (Wellcome Library, London).

20. Andreas Vesalius, De humani corporis fabrica, Basel, J. Oporinus, 1543.

Prima musculorum tabula (p. 170), woodcut (Wellcome Library, London).

21. Andreas Vesalius, Suorum de humani corporis fabrica librorum epitome, Basel, J. Oporinus, 1543. Figurae ad tabulam aptandam paratae . . . (fol. [N]r), woodcut (Wellcome Library, London).

22. Andreas Vesalius, Tabulae anatomicae sex, Venice, B. Vitalis, 1538.

Tabula I. Jecur. . . Generationis organa, woodcut (from Sir W.

Stirling-Maxwell (ed.), Tabulae anatomicae sex, London, 1874; photo:

Wellcome Library, London).

23. Andreas Vesalius, Tabulae anatomicae sex, Venice, B. Vitalis, 1538.

Tabula II. Venae cavae ... descriptio, woodcut (from Sir W.

Stirling-Maxwell (ed.), Tabulae anatomicae sex, London, 1874; photo:

Wellcome Library, London). 
24. Andreas Vesalius, Tabulae anatomicae sex, Venice, B. Vitalis, 1538. Tabula IV. Humani corporis ossa parte anteriori expressa, woodcut (from Sir W. Stirling-Maxwell (ed.), Tabulae anatomicae sex, London, 1874; photo: Wellcome Library, London).

25. Andreas Vesalius, Tabulae anatomicae sex, Venice, B. Vitalis, 1538.

Tabula V. Lateralis $\Sigma$ KE $\Lambda E T O Y$ figurae designatio, woodcut (from

Sir W. Stirling-Maxwell (ed.), Tabulae anatomicae sex, London, 1874;

photo: Wellcome Library, London).

26. Andreas Vesalius, Tabulae anatomicae sex, Venice, B. Vitalis, 1538.

Tabula VI. $\Sigma K E \Lambda E T O N$ a tergo delineatum, woodcut (from Sir W.

Stirling-Maxwell (ed.), Tabulae anatomicae sex, London, 1874; photo:

Wellcome Library, London).

27. Aegidius Macrolios (cut by the monogrammist V.H.), Cerebrum

animalis facultatis fons . . . , in reprint of Andreas Vesalius, Tabulae anatomicae sex, Cologne, L. Molendinus, 1539, woodcut (Wellcome Library, London).

28 and 29. Osteotome .i. [sic] ossium corporis humani divisio, ex Galeno praecipue collecta, Paris, C. Wechel, 1538, woodcuts (Collections of the Swedish Royal Library, Stockholm).

30. Johannes Dryander (ed.), Anatomia Mundini . . ., Marburg,

C. Egenolff, 1541. Urinary tract (fol. H1v), woodcut (Wellcome

Library, London).

31. Johannes Dryander (ed.), Anatomia Mundini . . , Marburg,

C. Egenolff, 1541. Intestines (fol. E3r), woodcut (Wellcome

Library, London).

32. Walter Hermann Ryff, Omnium humani corporis partium

descriptio . . . , Paris, J. de Gourmont, 1545. Prima figura viri . . .

(fol. A2r), woodcut (Wellcome Library, London).

33. Hans Wechtlin (or Wächtlin), Anatomia corporis humani,

Strasbourg, J. Schott, 1517, woodcut (Wellcome Library, London).

34. Walter Hermann Ryff, Omnium humani corporis partium descriptio ...., Strasbourg, Balthasar Beck (alias Pistor), 1541. Secunda figura anatomiae humani capitis (fol. b1v), woodcut (from W. H. Ryff, Omnium humani corporis partium descriptio . . . , facsimile, Basel, Sandoz, 1954; photo: Wellcome Library, London).

35. Johannes Dryander, Anatomia capitis humani, Marburg, E. Cervicornus, 1536. Anatomiae humani capitis figura secunda, (fol. B1r), woodcut

(Wellcome Library, London). 
36. Andreas Vesalius, Von des menschen Cörpers Anatomey, ein kurtzer aber vast nützer Ausszug . . . , Basel, J. Herpst (alias J. Oporinus), 1543. Inn diser letsten figur. . . , woodcut figures pasted together. (By permission of the Huntington Library, San Marino, CA.)

37. [H. Vogtherr], Auszlegung und beschreybung der Anatomi . . , Nuremberg, Hans Weygel, 1563. Title-page (The National Library of Medicine, Bethesda, MD).

38. [H. Vogtherr], Auszlegung und beschreybung der Anatomi . . , Nuremberg, Hans Weygel, 1563. Woodcut figure of the matrix and related text (The National Library of Medicine, Bethesda, MD).

39. Thomas Geminus, Compendiosa totius anatomie ... , London, J. Herford, 1545. De organis nutritioni . . dedicatis. Prima[-quarta] figura. (Plate following sig. F3), engraving (Wellcome Library, London).

40. Thomas Geminus, A figure for necessary lettyng off bloude, London, J. Herford, [c.1546], engraving (by permission of the Trustees of the National Library of Scotland, Edinburgh).

41. Johann Remmelin, Catoptrum microcosmicum . . , Augsburg, D. Franck, 1619. Title-page (Wellcome Library, London).

42. Figura infirmitatum, MS. Lat. 11229, fol. 31r (cliché Bibliothèque nationale de France, Paris).

43. Figura infirmitatum (also described as a "gravida figure"), MS. 1122, fol. 348r (by permission of the University Library, Leipzig; photo: from K. B. Roberts and J. D. W. Tomlinson, The fabric of the body, Oxford, 1992).

44. Anatomical figure. The arteries and the heart, Ms. Ashmole 399, fol. 19r (Bodleian Library, Oxford).

45. Skeletal figure, Ms. D.II.11, fol. 169v, University Library, Basel (from L. Choulant, History and bibliography of anatomic illustration, trans. and ed. M. Frank, Chicago, 1920; photo: Wellcome Library, London).

46. Pregnant anatomical figure. Mansur ibn Ilyas, Tashrih-i Mansuri.

Trent Ms. fol. 44r (property of Duke University Medical Center Library, History of Medicine Collections, Durham, NC).

47. Two anatomical figures (sagittal sections), Ms. X. 118 (Collections of the Swedish Royal Library, Stockholm).

48. Anatomical figure with flaps, Guido da Vigevano, Anathomia. MS. 334, fol. 264r, Musée Condé (from E. Wickersheimer (ed.), Anatomies de

* Mondino dei Luzzi et de Guido de Vigevano, Paris, 1926; photo:

Wellcome Library, London). 
49. Ein Contrafacter Todt ..., Strasbourg, J. Schott, 1517, woodcut (Wellcome Library, London).

50. Richard Helain, Anathomia ossium totius humani corporis, Leipzig, Wolfgang Stoeckel, 1501, woodcut (by permission of the British Library, London: shelfmark IA.22560(1)).

51. Skeleton, MS. fr. 19994, fol. 38v (cliché Bibliothèque nationale de France, Paris).

52. The city of London, as it was before the burning of St. Pauls steeple, [London, Gilles Godet, c. 1560], woodcut (Pepys Library, Magdalene College, Cambridge).

53. The good Hows-holder, [London, Paul Boulenger,] 1607, woodcut (Copyright British Museum, London).

54. Le divin philosophe, Antwerp, Sylvester van Parijs, [c.1545-50], woodcut (Printroom of the Museum Plantin-Moretus, Antwerp).

55. Le vieillard discrest, Lyon, Jean le Maistre and Antoine Volant, [mid-sixteenth century], woodcut (Museum Boijmans Van Beuningen, Rotterdam).

56. Edward the iiii. Edward the v. Richard the iii. from the series of woodcuts A brief abstract of the genealogie ... of all the kynges of England, [London, Gilles Godet, c. 1562], woodcut (by permission of the British Library, London: shelfmark G.6456).

57. Monogrammist PVD, Vvillem die eerste, Vvhillem rufus die ii. Henricus de eerste, from the series bearing the incipit Prologhe van allen den Coninghen in Enghelant ... [Antwerp] 1534, woodcut (by permission of the British Library, London: shelfmark C.39.1.2).

58. Howe Josephe is sett in great authoritie in Egypte, from the series of woodcuts The historie of Joseph, [London, Gilles Godet, c. 1565?], woodcut (cliché Bibliothèque nationale de France, Paris).

59. "Loo the prodigall childe wasteth ... ", from the series of woodcuts L'enfant prodigue, [London, Gilles Godet,] 1566, woodcut (cliché Bibliothèque nationale de France, Paris). 